\title{
De Zadig à Vautrin : sur le paradigme indiciaire chez Balzac
}

Andrea Del Lungo

\section{(2) OpenEdition}

\section{Journals}

\section{Édition électronique}

URL : http://journals.openedition.org/rief/250

DOI : 10.4000/rief.250

ISSN : 2240-7456

\section{Éditeur}

Seminario di filologia francese

\section{Référence électronique}

Andrea Del Lungo, "De Zadig à Vautrin : sur le paradigme indiciaire chez Balzac », Revue italienne d'études françaises [En ligne], 3 | 2013, mis en ligne le 15 décembre 2013, consulté le 01 mai 2019 URL : http://journals.openedition.org/rief/250 ; DOI : 10.4000/rief.250

Ce document a été généré automatiquement le 1 mai 2019.

\section{(c) (i) (9)}

Les contenus de la RIEF sont mis à disposition selon les termes de la Licence Creative Commons Attribution - Pas d'Utilisation Commerciale - Pas de Modification 4.0 International. 


\title{
De Zadig à Vautrin : sur le paradigme indiciaire chez Balzac
}

\author{
Andrea Del Lungo
}

1 La relation entre l'œuvre de Balzac et le siècle des Lumières se situe à deux niveaux complémentaires : celui des sources, et donc d'une influence de la littérature et de la philosophie du XVIII siècle sur la création de La Comédie humaine; et celui du siècle de Lumières en tant qu'horizon culturel et scientifique faisant l'objet d'une réévaluation dans la société post-révolutionnaire. Cette double relation est d'autant plus problématique que l'œuvre de Balzac modifie profondément le code romanesque et contribue à légitimer le genre même du roman, lui attribuant une fonction de connaissance du réel, de l'histoire et de la société qui fonde, selon Auerbach, un réalisme « moderne », en rupture avec les catégories esthétiques et idéologiques du passé.

2 Ce n'est pas un hasard si la question de l'héritage des Lumières dans l'œuvre de Balzac a été au cœur d'un débat qui a fait émerger des positions contradictoires dans l'histoire de la critique balzacienne. Je voudrais commencer par aborder ce point, en anticipant l'issue de la controverse : la critique actuelle semble en effet pencher vers l'hypothèse d'une rupture de continuité, c'est-à-dire d'une certaine autonomie du roman balzacien par rapport à l'héritage des Lumières et, plus généralement, du passé. J'essayerai alors de nuancer cette position par l'analyse de certaines formes de continuité, rapportées notamment aux principes de classifications qui fondent le réalisme. Dans une dernière partie, j'étudierai enfin la pertinence d'un fil conducteur particulier, celui du paradigme indiciaire décrit par Carlo Ginzburg, dont la généalogie littéraire remonte, entre autres, à Zadig de Voltaire; ce qui permettra d'avancer une hypothèse concernant les principes de classement propres au roman balzacien, qui relèvent d'une logique inductive, fondée moins sur l'analyse du type que sur celle de l'individu. 


\section{La controverse sur l'héritage des Lumières}

3 Notre histoire de la critique peut commencer par le rappel de la position de Curtius, qui affirme la profonde continuité de l'œuvre balzacienne avec la philosophie des Lumières, dans un essai magistral de 1933 consacré notamment à la notion d'énergie et à ses implications philosophiques et esthétiques, qui prône une vision antiromantique du roman balzacien. Cette position se développe en trois points : le premier est la relation de causalité, que Curtius élève à principe fondateur, «formule générale » qui investit aussi le domaine de la mystique, dont la généalogie remonte à Aristote pour arriver précisément à la philosophie des Lumières ${ }^{1}$.

4 Le deuxième point concerne la priorité assignée à l'intelligence dans l'interprétation du social, ce qui permet d'établir un lien direct entre la philosophie des Lumières et le positivisme :

Le processus dynamique de la société moderne doit être saisi, interprété, dirigé à la lumière de l'intelligence. Au début de la décennie de 1830, toutes ses réflexions sur les problèmes de son temps découlent de l'idée selon laquelle l'économie produit des valeurs de toute sorte et que l'intelligence et l'économie sont liées. Balzac est porté ici par le grand mouvement issu de la philosophie des Lumières au XVIII siècle, qui conduit au positivisme du XIXe siècle, pour trouver finalement dans le Saint-simonisme une expression particulièrement fertile. ${ }^{2}$

5 Le troisième point, sans doute le plus important, touche à la portée morale de l'œuvre : "La littérature du XVIII ${ }^{e}$ siècle s'est voulue éducatrice et réformatrice. Les grands écrivains des Lumières "livraient des batailles morales". Balzac leur rend hommage à tous. De tous les éléments historiques qui l'ont constitué, l'esprit du XVIII ${ }^{e}$ siècle est probablement le plus important pour son œuvre $»^{3}$. Curtius analyse ensuite l'influence de Montesquieu et de Diderot, pour conclure sur la définition d'un "universalisme des esprits » comme trait commun à la littérature du XVIII ${ }^{e}$ siècle et à l'œuvre de Balzac.

La critique d'inspiration marxiste, de Lukacs à Barbéris, ne parait pas remettre en cause le lien avec la philosophie des Lumières, au nom d'un matérialisme - certes renouvelé et anti-bourgeois - qui permet de soutenir la thèse déjà exprimée par Engels d'un Balzac révolutionnaire malgré lui, en dépit de ses positions explicitement réactionnaires. Mais ensuite, la vague structuraliste, prônant le principe de la décontextualisation historique, appliqué précisément à Balzac avec le $S / Z$ de Barthes (1970), escamote évidemment toute question d'héritage ou d'influence. Globalement, la critique balzacienne, à partir de cette époque, penche vers l'hypothèse d'une rupture de continuité par rapport au passé; l'enjeu étant de montrer la modernité du roman balzacien (le plus souvent contre la vision barthésienne du Balzac "lisible», et donc classique), il fallait à tout prix déraciner l'œuvre de son innutrition dix-huitiémiste, et la tourner vers le futur.

7 Ce n'est qu'en 1997 qu'un numéro de L'Année balzacienne revient sur la question grâce à une série d'articles sur Balzac et le XVIII ${ }^{e}$ siècle, parmi lesquels il faut citer celui de RenéAlexandre Courteix (auteur d'un ouvrage sur Balzac et la révolution française), qui a le mérite de proposer une analyse plus fine du parcours intellectuel de l'auteur de $L a$ Comédie humaine. Il est nécessaire de rappeler d'abord une donnée biographique: la philosophie des Lumières a eu une importance fondamentale dans la formation du jeune Balzac, dont le père, François-Bernard, rédigea plusieurs opuscules traitant de questions d'hygiène, de criminalité, de réformes sociales, exprimant une vision matérialiste, 
largement redevable de la lecture de Voltaire, et surtout de l'Encyclopédie. Courteix affirme que Balzac aurait hérité des Lumières, par son père, « une conception matérialiste et mécaniste de l'homme et de la nature, un rationalisme incroyant $»^{4}$. Les romans de jeunesse de Balzac, inspirés du point de vue littéraire aux modèles du roman gothique, développent néanmoins, selon Courteix, des arguments rationalistes voués à dénoncer l'obscurantisme du passé. Mais ensuite - reconnaît le critique - Balzac affichera des thèses de plus en plus contraires à la philosophie des Lumières, allant jusqu'à récuser le voltairianisme, notamment en ce qui concerne le libre arbitre, considéré comme un facteur de désordre social.

8 Selon une opinion courante à l'époque, l'œuvre des philosophes serait tenue pour responsable de la Révolution française, comme on peut lire dans un passage du Catéchisme social de 1840 : «Toutes le fois que les destructeurs de société ont voulu miner un état de choses quelconque, ils ont toujours été précédés de sophistes habiles qui ont essayé d'établir table rase. Ils ont pour renverser le pouvoir établi créé des préjugés philosophiques: le libre arbitre, liberté de conscience, la liberté politique, qui se sont traduits par des révolutions $»^{5}$. Dans son essai, Courteix résume en trois points le caractère néfaste qu'aurait eu la philosophie des Lumières selon Balzac: le premier « $\mathrm{a}$ trait à l'esprit de discussion, de contestation et d'opposition $\|^{6}$ engendré par la mise en cause permanente de l'autorité et des valeurs; le deuxième concerne l'anticléricalisme, qui mine le caractère fondateur de la religion comme facteur d'ordre social ; le troisième tient de la dégradation des structures familiales, conséquence d'un individualisme hérité des Lumières, et que « la Révolution a fait passer dans la loi, puis dans les mœurs »?

Pour nuancer quelque peu la vision que donne Courteix d'un Balzac contrerévolutionnaire, on pourrait alors ajouter une autre explication du caractère néfaste de la philosophie des Lumières, située cette fois au niveau de l'analyse de la reconstitution d'un imaginaire culturel: à l'époque de Balzac, la bourgeoisie s'était en effet approprié l'héritage philosophique des Lumières, en l'investissant de toute sa bêtise et en en aplatissant la portée idéologique sur ce mythe positiviste du progrès qui devint rapidement dominant dans la culture du siècle, parallèlement à l'ascension sociale et politique de cette même bourgeoisie. Balzac n'hésite pas à porter un regard critique sur un tel processus historique (qui s'incarnera, chez Flaubert, dans la figure emblématique du pharmacien Homais), notamment par le biais de l'ironie : ainsi, dans Illusions perdues, Lousteau conseille à Lucien, aspirant journaliste, de placer dans son article « un éloge de Voltaire, Rousseau, Diderot, Montesquieu et Buffon $»^{8}$, afin de complaire au lecteur bourgeois.

\section{Continuité ou fracture?}

10 Pour essayer de donner une lecture plus objective par rapport à cette controverse historique qui oppose un Balzac révolutionnaire (matérialiste et rationaliste) à un Balzac réactionnaire (idéaliste et spiritualiste), on pourrait alors analyser les références explicites aux philosophes que présente l'œuvre balzacienne. Or, les noms de Voltaire, Montesquieu, Diderot et Rousseau ${ }^{9}$ reviennent souvent dans La Comédie humaine; mais ces citations se révèlent en réalité peu substantielles, dans la mesure où elles renvoient généralement moins à la philosophie, à la pensée ou à l'œuvre de ces auteurs, qu'à la figuration du génie qu'ils incarnent. 
11 Le cas de Voltaire est, de ce point de vue, emblématique d'un emploi qui relève quasi exclusivement de l'antonomase. Parmi les nombreux exemples, il suffit de songer à la définition de l'Arétin comme "le Voltaire de son temps" ou "de son siècle", qui intervient à plusieurs reprises ${ }^{10}$; ou encore au syntagme " esprit voltairien ", fréquent chez Balzac, ainsi qu'à une variation de celui-ci dans un commentaire du narrateur à propos du personnage de Louis Lambert : « Pour exprimer en deux mots son talent, il eût écrit Zadig aussi spirituellement que l'écrivit Voltaire ${ }^{11}$. Enfin, le nom du philosophe revient comme un leitmotiv dans les célèbres énumérations balzaciennes retraçant la généalogie de l'esprit et du génie, au nombre d'une quinzaine dans La Comédie humaine: "Il y a dans tous les temps un homme de génie qui se fait le secrétaire de son époque: Homère, Aristote, Tacite, Shakespeare, l'Arétin, Machiavel, Rabelais, Bacon, Molière, Voltaire, ont tenu la plume sous la dictée de leurs siècles $»^{12}$.

12 L'emploi de l'antonomase semble ainsi vider la référence de toute portée conceptuelle; pour preuve, l'« Avant-propos » de La Comédie humaine, texte qui expose la généalogie philosophique et scientifique de l'œuvre, ne présente aucun renvoi aux philosophes, exception faite d'une note critique contre Rousseau à propos du rôle de la société comme facteur de dépravation de l'homme.

Deux constats émergent de cette rapide étude des références balzaciennes, fondée sur l'œuvre mais aussi sur l'«Avant-propos" et les préfaces. Le premier est que la philosophie balzacienne se nourrit d'apports extrêmement éclectiques: du siècle des Lumières, Balzac hérite le concept de l'unité organique de l'homme et des êtres vivants, principe fondamental des théories de Buffon; auquel s'ajoute cependant un concept d'unité de l'univers qui renvoie plutôt à la monadologie de Leibniz (souvent employé comme métaphore de l'œuvre d'art), ainsi qu'un concept d'unité mystique entre les deux parts de l'homme (intérieur et extérieur) dans sa relation au divin, que l'on doit à Swedenborg. De toute évidence, un tel éclectisme empêche de concevoir des éléments de continuité globale par rapport à n'importe quelle tradition philosophique.

Le second constat est encore plus significatif : le roman balzacien semble se fonder sur des savoirs essentiellement contemporains ${ }^{13}$ : le modèle essentiel, qui sert entre autres à définir la tâche du romancier, est celui de l'anatomie comparée, auquel Balzac renvoie en épousant d'abord les thèses de Cuvier (partisan de l'immutabilité des espèces), puis de son adversaire Geoffroy Saint-Hilaire, qui soutenait un point de vue évolutionniste ${ }^{14}$; mais Balzac est aussi influencé par la physiognomonie, ainsi que par la phrénologie de Gall et Lavater, discipline naissant au début du siècle. Cette référence à des savoirs contemporains semble donc corroborer l'hypothèse d'une fracture substantielle par rapport à des paradigmes scientifiques et à des théories philosophiques du passé.

Je voudrais alors essayer de définir certains liens susceptibles d'articuler le roman balzacien à la philosophie des Lumières, concernant notamment les critères d'analyse et les principes de classement $d u$ réel, éléments fondateurs d'une œuvre qui vise non seulement à reproduire toutes les facettes de la réalité mais aussi, d'un point de vue idéologique, à rétablir des méthodes de différenciation au sein d'une société postrévolutionnaire particulièrement mouvante et indifférenciée, aux yeux de Balzac. Nostalgique de l'Ancien régime et de la fixité de l'état social précédent la Révolution, Balzac analyse le nouvel état social au moyen d'un paradigme typologique, dont le but est d'ordonner le chaos en rétablissant une hiérarchie. Telle est la leçon de l'« Avantpropos ", et notamment de la comparaison avec la zoologie qui renvoie à Buffon : 
Il n'y a qu'un animal. Le créateur ne s'est servi que d'un seul et même patron pour tous les êtres organisés. L'animal est un principe qui prend sa forme extérieure, ou, pour parler plus exactement, les différences de sa forme, dans les milieux où il est appelé à se développer. Les Espèces Zoologiques résultent de ces différences. ${ }^{15}$

La théorie du milieu, élément fondamental qui influence et détermine l'espèce, ici formulée à propos du règle animal, se trouve ensuite appliqué à l'homme :

Pénétré de ce système bien avant les débats auxquels il a donné lieu, je vis que, sous ce rapport, la Société ressemblait à la Nature. La Société ne fait-elle pas de l'homme, suivant les milieux où son action se déploie, autant d'hommes différents qu'il y a de variétés en zoologie? Les différences entre un soldat, un ouvrier, un administrateur, un avocat, un oisif, un savant, un homme d'état, un commerçant, un marin, un poète, un pauvre, un prêtre, sont, quoique plus difficiles à saisir, aussi considérables que celles qui distinguent le loup, le lion, l'âne, le corbeau, le requin, le veau marin, la brebis, etc. Il a donc existé, il existera donc de tout temps des Espèces Sociales comme il $\mathrm{y}$ a des Espèces Zoologiques. Si Buffon a fait un magnifique ouvrage en essayant de représenter dans un livre l'ensemble de la zoologie, n'y avait-il pas une œuvre de ce genre à faire pour la Société ? ${ }^{16}$

Le procédé auquel recourt Balzac est celui de la typisation, c'est-à-dire de la définition, à partir d'un regard général et panoramique sur la société humaine, des différents types humains qui la composent. Ce principe de classification déterministe, veiné d'un certain positivisme, ne paraît pas très éloigné du modèle de l'Encyclopédie, dont l'ambition était de définir et de classer non seulement le savoir, mais aussi les arts et métiers. Il serait donc possible de supposer un héritage dix-huitiémiste en observant la vocation encyclopédique de l'entreprise balzacienne, qui trouve sa réalisation dans des morceaux de littérature panoramique intégrés au romans de La Comédie humaine. Cependant, ce procédé de typisation relève souvent de la caricature, par la mise en exergue des lieux communs qui définissent les différents types humains, comme en témoigne la relation entre le texte et l'image: dans les recueils de littérature panoramique auxquels participa Balzac (Les Français peints par eux-mêmes, par exemple), la description de chaque type est en effet accompagné d'une illustration, souvent caricaturale ; il est également utile de rappeler que la première illustration de La Comédie humaine dans l'édition Furne de 1842 représente le figure d'un commerçant (le père Guillaume, patron de la Maison du chat-qui-pelote), qui résume précisément les traits typiques de cette « espèce » sociale.

Manifestement, le principe de classification déterministe ne fonctionne pas dans l'œuvre ; ou, plus précisément, il risque de réduire la complexité du réel à une représentation simpliste (comme dans le cas de l'opposition entre l'artiste et le bourgeois), immuable, voire caricaturale. La limite du principe réside dans son caractère déductif : procédant du général au particulier, c'est-à-dire en attribuant des traits typiques à l'individu, la classification déterministe ne pourrait pas répondre à l'exigence d'une différenciation nécessaire aux yeux de l'auteur. Tel est, me semble-t-il, le sens de la célèbre préface d'Une fille d'Ève (1839), dans laquelle Balzac exprime son angoisse devant un état social égalitaire, et par conséquent indistinct :

Autrefois tout était simplifié par les institutions monarchiques; les caractères étaient tranchés : un bourgeois, marchand ou artisan, un noble entièrement libre, un paysan esclave, voilà l'ancienne société de l'Europe; (elle prêtait peu aux incidents du roman. Aussi voyez ce que fut le roman jusqu'au règne de Louis XV). Aujourd'hui, l'Égalité produit en France des nuances infinies. Jadis, la caste donnait à chacun une physionomie qui dominait l'individu ; aujourd'hui, l'individu ne tient sa physionomie que de lui-même ${ }^{17}$. 
19 La fin de la réflexion introduit un aspect fondamental: afin de pouvoir décrire les «nuances infinies» permettant de différencier la nouvelle société contemporaine, le point de départ doit être l'individu, considéré moins dans son appartenance à une espèce ou à un type, que dans sa singularité.

\section{Un paradigme inductif}

20 Un nouveau fil conducteur - celui annoncé dans mon titre - permet alors de lier le roman balzacien à un passé, suivant la généalogie de ce modèle de connaissance que Carlo Ginzburg définit le « paradigme indiciaire », qui présente la particularité, par rapport aux modèles précédents, d'être à la fois qualitatif et inductif. Dans son article fondateur ${ }^{18}$, Ginzburg situe l'émergence du paradigme indiciaire dans la seconde moitié du XIX ${ }^{\mathrm{e}}$ siècle, époque où se développent un ensemble de disciplines (phrénologie, physiognomonie, anthropométrie, graphologie) fondées sur l'analyse des signes concrets, extérieurs et visibles. Pour définir ce paradigme inédit, Ginzburg porte le triple exemple de l'historien de l'art Morelli, proposant une nouvelle méthode pour l'attribution des tableaux fondée sur « l'examen des détails les plus négligeables où l'influence des caractéristiques de l'école à laquelle le peintre appartenait est moins marquée - ce qui est le cas des lobes des oreilles des ongles, de la forme des doigts et des orteils ${ }^{19}{ }^{19}$ du detective Sherlock Holmes, «qui découvre l'auteur du délit en se fondant sur des indices qui échappent à la plupart des gens $»^{20}$; et du père de la psychanalyse, Freud, à qui revient "l'idée d'une méthode d'interprétation s'appuyant sur les déchets, sur les données marginales considérés comme révélateurs $»^{21}$. Ainsi, les signes picturaux, les indices policiers et les symptômes sont-ils réunis dans un paradigme qui, à partir de «traces parfois infinitésimales, permet d'appréhender une réalité plus profonde, qu'il serait impossible de saisir par d'autres moyens $»^{22}$.

21 Dans la deuxième partie de son essai, Ginzburg retrace la généalogie du paradigme, dont l'origine est en réalité ancienne, et indique quelques références littéraires : le premier est un récit oral appartenant à la tradition du Moyen-Orient, qui raconte l'histoire de trois frères capables, à partir d'indices minimes, de reconstituer l'aspect d'un animal qu'ils n'avaient jamais vu. Cette trame narrative (qui intègre l'explication de la méthode, par laquelle les frères sont acquittés de l'accusation de vol de l'animal), inspirera le troisième chapitre de Zadig de Voltaire ${ }^{23}$, dans lequel le héros décrit deux animaux, une chienne et un cheval, grâce à l'analyse des traces laissées sur le sol. L'historien rappelle qu'au cours du XIX ${ }^{e}$ siècle on définissait comme " méthode de Zadig » le procédé commun à l'histoire et à l'archéologie, mais aussi à d'autres disciplines des sciences naturelles, consistant à «faire des prophéties rétrospectives» sur la base d'un renversement de la causalité : «Lorsque les causes ne sont pas reproductibles, il ne reste qu'à les induire des effets ${ }^{24}$.

Or, Ginzburg ne développe évidemment pas cette généalogie littéraire (qui n'est pas son sujet principal), et se limite à remarquer que dans les explications de Zadig on peut trouver «l'embryon du roman policier », mentionnant ensuite les textes canoniques de Poe, Gaboriau e Conan Doyle. Balzac, souvent considéré comme un ancêtre du genre policier, n'est pas cité par l'historien, de manière à vrai dire un peu surprenante : il me semble en effet que le roman balzacien joue un rôle fondamental dans cette filiation, que j'essayerai de résumer en trois points. 
23 En premier lieu, la méthode de Zadig, selon la définition finale qu'en donne Ginzburg («Lorsque les causes ne sont pas reproductibles, il ne reste qu'à les induire des effets ») fonde l'architecture globale de La Comédie humaine, dans laquelle l'analyse des «effets sociaux » - objet principal, selon l'auteur, des «Études de mœurs », qui sont la partie la plus développée de l'œuvre - précède celle des «causes sociales» et des "principes sociaux », objet des «Études philosophiques» et des «Études analytiques»: de toute évidence, les trois niveaux qui articulent l'œuvre balzacienne relèvent d'une logique inductive que le romancier définit dès 1834, dans une célèbre lettre à Madame Hanska annonçant les critères de composition de l'ensemble ${ }^{25}$.

Le deuxième élément qui prouve le rôle du roman balzacien dans la généalogie du paradigme indiciaire (et qui corrobore aussi l'hypothèse selon laquelle le roman aurait élaboré ce paradigme même avant sa généralisation aux sciences naturelle ${ }^{26}$ ) concerne la prépondérance absolue des signes concrets et des détails indiciaires dans le roman de l'époque, ainsi que la relative nouveauté historique d'une telle prolifération dans l'histoire du genre. La description réaliste abonde en détails et relève de cette " passion de la concrétude - évoquée par Jacques Neefs à propos du roman balzacien - qui donne à la présence de toute chose valeur de signe $\aleph^{27}$. On connaît d'ailleurs l'importance capitale du détail chez Balzac, non seulement dans l'œuvre romanesque mais aussi dans la poétique qu'affirme l'auteur de La Comédie humaine, « croyant fermement que les détails seuls constitueront désormais le mérite des ouvrages improprement appelés Romans ${ }^{28}$. Le détail se trouve ainsi placé au cœur d'une activité herméneutique qui permet au romancier - analyste de la société et historien des mœurs, dans la vision balzacienne - de reconstruire le tout, par analogie, à partir du déchiffrement des données marginales: scories, restes, fragments, reliques. Tel est d'ailleurs le rôle, par exemple, de la description architecturale sur laquelle s'ouvrent plusieurs romans balzaciens ( $L a$ Maison du chat-qui-pelote, La Recherche de l'absolu, Eugénie Grandet, etc.): exposer des détails comme signes révélateurs du monde, aussi bien de la sphère sociale que de la sphère privée ; et l'observateur peut ainsi « reconstruire les nations ou les individus dans toute la vérité de leurs habitudes, d'après les restes de leurs monuments publics ou par l'examen de leurs reliques domestiques $»^{29}$. Le détail se trouve donc sur-semantisé, enflé de signification, investi d'un pouvoir connotatif qui en fait le signe par excellence dans la quête d'une totalité (comme dans les exemples cités), mais aussi dans la définition de l'identité individuelle.

Le troisième aspect, qui confirme la pertinence du paradigme indiciaire et de la logique inductive, concerne la classification identitaire qu'opère le roman balzacien, fondée en réalité moins sur l'analyse du type que sur celle de l'individu. Sur la scène du roman, le type semble occuper l'arrière-plan, alors qu'au premier plan se dresse le véritable héros romanesque : il s'agit le plus souvent, dans l'univers balzacien, d'un être exceptionnel, qui échappe précisément à la typologie sociale, et qui affirme à la fois le pouvoir et la singularité d'un individu hors-norme, auquel il est permis de traverser les classements instaurés par le roman lui-même. Le plus célèbre personnage balzacien, Vautrin, en fournit sans doute le meilleur exemple : marqué par la société comme escroc, voleur et criminel mais, heureusement pour lui, non pas marqué au fer, la pratique ayant disparu depuis peu -, sa trajectoire existentielle de l'ordre du déguisement perpétuel, que permet précisément l'absence de marque corporelle, le conduira au rôle de chef de la police. Pour donner un autre exemple, dans La Fille aux yeux d'or, après le tableau sociologique et normatif des physiologies parisiennes du début du texte, qui classe la société en cinq 
sphères d'appartenance (ou plutôt six, parce que la première sphère est double, regroupant les ouvriers et les petits merciers), l'histoire se focalise sur deux personnages inclassables : de Marsay, défini dès le début comme un être d'exception, qui ne rentre pas dans la typologie sociale que le narrateur vient d'établir; et Paquita, dont l'étrangeté absolue (du point de vue de l'origine, de l'identité, de la sexualité) contribue à mettre en question la classification elle-même.

Bref, le roman se concentre davantage sur les indices qui différencient et qui démarquent l'individu de son espèce, plutôt que sur les signes d'appartenance. Cette logique indiciaire investit aussi le rôle du narrateur : à l'instar de plusieurs personnages d'artistes ou de visionnaires, le narrateur balzacien est doté d'un don de seconde vue lui permettant de déchiffrer les signes que les autres ne voient pas. Grâce à ce don, il peut percer cette histoire secrète qui est l'objet de la leçon d'histoire que Vautrin, déguisé en abbé espagnol, donne au jeune Lucien de Rubempré, à la fin d'Illusions perdues, et que l'on peut lire comme une devise du roman: «Il y a deux Histoires : l'Histoire officielle, menteuse qu'on enseigne, l'Histoire ad usum delphini ; puis l'Histoire secrète, ou sont les véritables causes des événements, une Histoire honteuse $\star^{30}$. Tel serait alors le rôle du narrateur balzacien : dévoiler, dans la description du réel, ce qui reste caché, secret, invisible à celui qui n'est pas initié à l'herméneutique indiciaire.

Pour conclure, revenons à la question qui concerne la relation du roman balzacien avec le passé : continuité ou fracture? La réflexion que j'ai développée dans cet article n'aura sans doute pas permis de dénouer la problématique. J'ai cependant essayé de montrer quelques éléments d'une continuité possible, qui acquiert sa pertinence à condition d'entendre le concept d'héritage dans son acception la plus vaste: moins en termes d'influence, de source ou d'intertexte explicite, que comme un horizon imaginaire et culturel, fruit de la perception d'un passé qui devient présent. Dans le cas de Balzac, il me semble que la fracture esthétique par rapport aux modèles romanesques précédents, qui se trouvent profondément renouvelés, s'accompagne d'une forme de continuité dans les principes et dans les méthodes de l'analyse du réel, suivant la généalogie du paradigme indiciaire.

À l'instar de Zadig, l'écrivain se fait alors observateur des indices, en quête de leur déchiffrement. Au fond, Balzac nous le suggère, en même temps qu'il définit le dilemme de l'écrivain, obligé d'articuler l'invention et l'observation:

L'observation des phénomènes relatifs à l'homme, l'art qui doit en saisir les mouvements les plus cachés, l'étude du peu que cet être privilégié laisse involontairement deviner de sa conscience, exigent une somme de génie. [...] L'écrivain, chargé de répandre les lumières qui brillent sur les hauts lieux, doit donner à son œuvre un corps littéraire, et faire lire avec intérêt les doctrines les plus ardues, et parer la science. Il se trouve donc sans cesse dominé par la forme, par la poésie, et par les accessoires de l'art. Être un grand écrivain et un grand observateur, Jean-Jacques et le Bureau des Longitudes, tel est le problème; problème insoluble ${ }^{31}$. 


\section{NOTES}

1. E. R. Curtius, Balzac, Paris, Grasset, 1933 (éd. or. 1923), p. 54. Nous reviendrons sur ce point.

2. Ibid., p. 230.

3. Ibid., p. 314.

4. R.-A. Courteix, «Les Philosophes et la Révolution française dans l'œuvre de Balzac », L'Année balzacienne, 1997, p. 162.

5. H. de Balzac, Catéchisme social, dans Euvres complètes, Paris, Club de l'Honnête Homme, 1955-1963, t. XXVII, p. 511.

6. R.-A. Courteix, "Les Philosophes et la Révolution française dans l'œuvre de Balzac », cit., p. 166.

7. Ibid., p. 168.

8. H. de Balzac, Illusions perdues, dans La Comédie humaine, Paris, Gallimard, «Bibliothèque de la Pléiade ", t. V, 1976, p. 443.

9. Vis-à-vis duquel Balzac a une position ambivalente, que décrit R. Trousson dans son ouvrage Balzac disciple et juge de Jean-Jacques Rousseau, Genève, Droz, 1983.

10. Voir, dans l'édition citée de la "Bibliothèque de la Pléiade", Illusions perdues, t. V, p. 121, et Sur Catherine de Médicis, t. X, p. 169 et 200.

11. H. de Balzac, Louis Lambert, dans La Comédie humaine, cit., t. X, p. 640.

12. H. de Balzac, Théorie de la démarche, dans La Comédie humaine, cit., t. XI, p. 278.

13. J'emploie cet adjectif dans son acception temporellement étendue, qui renvoie généralement à une génération: en ce qui concerne Balzac, dont le premier roman rattaché à La Comédie humaine date de 1829, le « contemporain » correspondrait au début du XIX ${ }^{\mathrm{e}}$ siècle.

14. Voir les commentaires éclairants de S. Vachon dans ses notes à l'« Avant-propos » dans Balzac. Écrits sur le roman, Paris, Le livre de Poche, 2000, p. 278-279.

15. H. de Balzac, « Avant-propos » de La Comédie humaine, cit., t. I, p. 1.

16. Ibid.

17. H. de Balzac, préface à Une fille d’Ève, dans La Comédie humaine, cit. t. II, p. 263.

18. L'article parut en italien dans le volume collectif Crisi della ragione. Nuovi modelli nel rapporto tra sapere e attività umane (Torino, Einaudi, 1979) sous le titre «Spie. Radici di un paradigma indiziario »; il fut traduit en français dans la revue Le Débat, 6, 1980, sous le titre « Signes, traces, pistes. Racines d'un paradigme de l'indice ». Les citations suivantes renvoient à cette version.

19. C. Ginzburg, «Signes, traces, pistes. Racines d'un paradigme de l'indice », cit., p. 4.

20. Ibid., p. 7.

21. Ibid., p. 11.

22. Ibid., p. 12.

23. Ginzburg précise que Voltaire avait pu lire une version du conte oriental dans le recueil de nouvelles Peregrinaggio di tre giovani figliuoli del re di Serendippo, traduit en français au XVIII ${ }^{\mathrm{e}}$ siècle (Ibid., p. 32).

24. Ibid., p. 33.

25. On peut lire ce texte fondateur dans l'anthologie Écrits sur le roman, déjà citée précédemment, p. 81-85.

26. Sur ce point, je me permets de renvoyer à mon article «Temps du signe, signes du temps. Quelques pistes pour l'étude du concept de signe dans le roman du XIX ${ }^{\mathrm{e}}$ siècle ", dans Le Roman du signe. Herméneutique et fiction au XIX ${ }^{e}$ siècle, sous la direction d'A. Del Lungo et B. Lyon-Caen, SaintDenis, PUV, 2007. 
27. J. Neefs, «Les trois étages du mimétique dans La Comédie humaine», dans Balzac. CEuvres complètes. Le «moment » de La Comédie humaine, sous la direction de Cl. Duchet et I. Tournier, SaintDenis, PUV, 1993, p. 155.

28. H. de Balzac, « Note » de la première édition des Scènes de la vie privée (1830), reproduite dans l'édition de la « Bibliothèque de la Pléiade » de La Comédie humaine, cit., t. I, p. 1174-1175.

29. H. de Balzac, La Recherche de l'absolu, dans La Comédie humaine, cit., t. X, p. 657.

30. H. de Balzac, Illusions perdues, cit., p. 695.

31. H. de Balzac, Théorie de la démarche, dans La Comédie humaine, cit., t. XI, p. 277.

INDEX

Mots-clés : Lumières, romantisme, Voltaire, Balzac (Honoré de), paradigme indiciaire, Comédie humaine, Ginzburg (Carlo), méthodes de classification, principe inductif, analyse de l'individu 